\title{
Vascularização arterial da porção torácica do timo em suínos
}

\section{Arterial vascularization of the thoracic pars of the thymus in pigs}

Sérgio Salazar DRUMMOND; ${ }^{1}$ Pedro Primo BOMBONATO²; Frederico Ozanan Carneiro e SILVA'; Renato Souto SEVERINO ${ }^{1}$; André Luis Quagliato SANTOS ${ }^{1}$; Arani Nanci Bomfim MARIANA ${ }^{2}$

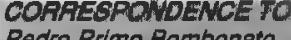
Pedro Primo Bombonato Departamento de Cirurgla Faculdado do Medlcina Veterinárla - Zootecnia da USP Av. Prof. Dr. Oriando Marques do Paiva, 87 - Clidade Universitária Paiva, 87 - Cldade Universitarit 05508-900 - SaO Paulo - SP - Brasil

1 - Faculahe de Madldne Veterinaria Universidade Foderal de Uberlandia - MG

\section{RESUMO}

Estudaram-se, em 30 fetos e recém-natos de suínos sem raça definida, mediante injeção de solução de Neoprene Látex 450 corado e posterior dissecação, a origem, o número e a ordenação dos vasos tímicos. A porção torácica do timo mostrou-se irrigada por ramos diretos e indiretos oriundos do tronco braquiocefálico, das aa. subclávias, torácicas internas, cervicais superficiais e do tronco tireocervical. Independentemente da origem e tipo, o número total de colaterais variou de 2 a 9, com maior freqüência de 3 .

UNITERMOS: Anatomia; Porcos; Timo; Vascularização; Artérias.

\section{INTRODUÇÃO}

A s significativas similares biológicas, estruturais e nutricionais do suíno com o ser humano levaram-nos, em anos recentes, a transformá-lo em elemento de escolha de muitas pesquisas na área biomédica. As questôes relativas à morfologia e à função tímica não estão totalmente esclarecidas, apesar dos notáveis avanços nos estudos e experimentos sobre este órgão.

Assim, estudos sobre a irrigação do timo em outras espécies animais têm sido realizados. Entretanto, especificamente sobre suínos notamos na literatura apenas os trabalhos de Daschinger $^{4}$ (1978) e Wustinger; Pospieszny ${ }^{19}$ (1984), que se mostram genéricos, o que nos motivou a desenvolver, nesta oportunidade, trabalho objetivando melhor conhecimento da vascularização arterial deste órgão, em particular a origem, o número e ordenação dos vasos destinados à porção torácica do timo.

\section{MATERIAL E MÉTODO}

Para a realização deste trabalho, utilizamos 30 suínos SRD, sendo 17 machos e 13 fêmeas, fetos e recém-natos em diferentes estágios de desenvolvimento e apresentando o comprimento variável de 17,3 a $28,3 \mathrm{~cm}$, medidas estas tomadas a partir da crista nucal à articulação entre a última vértebra sacral e a primeira vértebra caudal.

O material obtido quando da ocorrência de abortos ou mortes naturais, junto a criadores no Município de Uberlândia-
MG, teve injetada a aorta, na sua porção torácica, com solução aquosa de Neoprene Látex 450 corado com pigmento específico (Du Pont do Brasil S.A. - Indústria Química).

Posteriormente, as peças foram fixadas em solução aquosa de formol a $10 \%$ durante um período mínimo de 48 horas, para ulterior dissecação das artérias endereçadas ao timo, com auxílio de lente de aumento (Aumento 10x). A porção torácica foi estabelecida usando-se como ponto de reparo a margem cranial do primeiro par de costelas.

A nomenclatura utilizada na denominação dos vasos e do timo foi indicada pelo Comitê Internacional de Nomenclatura Anatômica Veterinária (Nomina Anatomica Veterinaria ${ }^{14}$, 1983).

A análise estatística aplicada aos resultados foi executada com auxílio do teste do qui-quadrado, com nível de significância de $5 \%$ e testada sua correlação pelo Teste de Correlação de Pearson, com nível de significância de $1 \%$, todos executados em microcomputador Microtec Mf88, por meio de programa intitulado "Statgraphics - Statistical Graphics System" - STSC, Inc, and Statistical Graphics Corporation, número de série 122467, versão 2.6 .

\section{RESULTADO}

1. Origem dos vasos tímicos (Tab. 1)

A parte torácica do timo de suínos é irrigada 29 vezes $(96,66 \% \pm 3,3)$ por ramos oriundos das aa. torácica interna esquerda e direita, aa. subclávia esquerda e direita, tronco braquiocefálico e a. cervical superficial esquerda. Esta parte 
DRUMMOND, S.S.; BOMBONATO, P.P.; SILVA, F.O.C.; SEVERINO, R.S.; SANTOS, A.L.Q.; MARIANA, A.N.B. Vascularizaçāo arterial da porção torácica do timo em suínos. Braz. J. vet. Res. anim. Sci. São Paulo, v.33, n.4, p.214-219, 1996

tímica não foi por nós identificada em $3,33 \% \pm 3,3$.

Em 26 peças $(86,66 \% \pm 6,2)$, o suprimento sanguíneo da supracitada parte tímica é realizado por número variável de ramos, sempre com a participação da a. torácica interna esquerda, aos quais se associam ramos, ora da a. torácica interna direita - $16,66 \% \pm 6,8$; ora da a. subclávia esquerda $10,0 \% \pm 5,5$; ora do tronco braquiocefálico $-6,66 \% \pm 4,5$; ou ainda em conjunto com as aa. torácica interna direita e subclávia esquerda - $16,66 \% \pm 6,8$; ou com as aa. torácica interna direita e tronco braquiocefálico - $10,0 \% \pm 5,5$; ou com as aa. torácica interna direita e subclávia direita $-6,66 \% \pm 4,5$; ou com as aa. subclávia esquerda e tronco braquiocefálico $3,33 \% \pm 3,3$; ou com as aa. tronco braquiocefálico, subclávia esquerda e torácica interna direita $-6,66 \% \pm 4,5$; ou com as aa. subclávia esquerda e tronco braquiocefálico - 3,33\% $\pm 3,3$; ou com as aa. tronco braquiocefálico, subclávia esquerda e torácica interna direita $-6,66 \% \pm 4,5$; ou com as aa. torácica interna direita, subclávia direita e tronco braquiocefálico $3,33 \% \pm 3,3$ ou com as aa. subclávia esquerda, torácica interna direita e tronco tireocervical $-3,33 \% \pm 3,3$; ou com as aa. torácica interna direita, subclávia esquerda, subclávia direita e cervical superficial esquerda - 3,33\% $\pm 3,3$. Nos 3 casos restantes $(10,0 \% \pm 5,5)$, a vascularização arterial ocorre, exclusivamente, por ramos da a. torácica interna esquerda (Fig. 1).

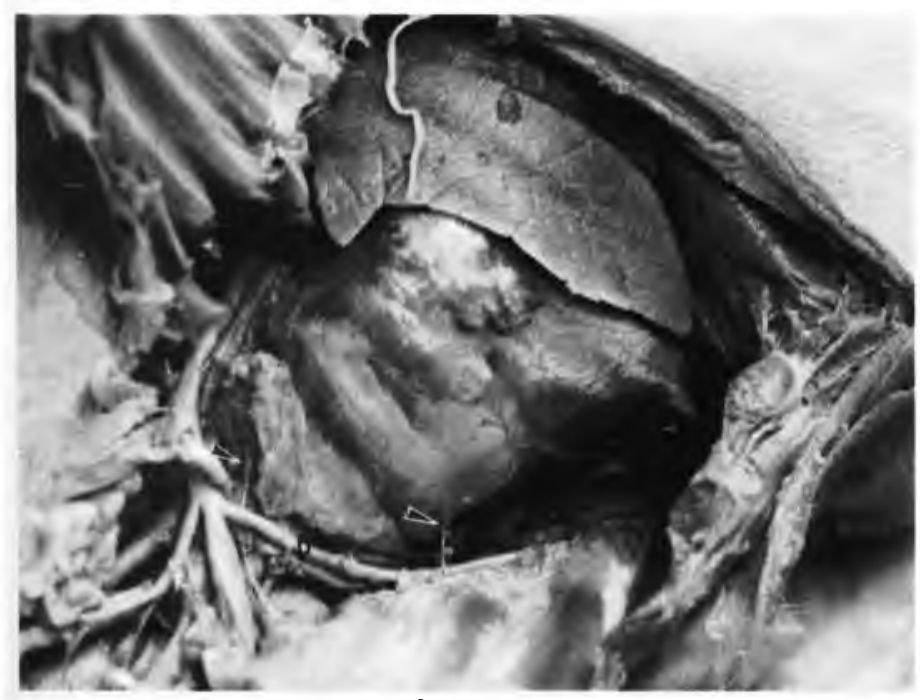

Figura 1

Fotografia da porção torácica do timo, em suínos SRD, mostrando dois ramos diretos (setas) oriundos da a. torácica interna esquerda (b). (Razão de ampliação $2 X$ ).

\section{Tipo, número de vasos na origem e quando penetram no} timo (Tab. 1)

Ao analisarmos o tipo (direto ou indireto) e o número de vasos, na origem e quando penetram no timo, observamos que quando ocorre a associação entre as aa. torácica interna esquerda e torácica interna direita em $16,66 \% \pm 6,8$, cada vaso emite respectivamente, em $6,66 \% \pm 4,5$ dos casos, dois ramos diretos e indivisos e um ramo direto e indiviso; em $3,33 \% \pm 3,3$ a a. torácica interna esquerda contribui com dois ramos diretos e a a. torácica interna direita com um ramo indireto, ambos indivisos; em $3,33 \% \pm 3,3$ a a. torácica interna esquerda emite dois ramos diretos e indivisos e um direito que se divide em quatro ramos, e a a. torácica interna direita envia um ramo direto e um indireto e indivisos; em $3,33 \% \pm 3,3$ a a. torácica interna esquerda contribui com três ramos diretos, enquanto a a. torácica interna direita, com um ramo direto e um indireto, todos indivisos.

Quando a combinação ocorre entre as aa. torácica interna esquerda e subclávia esquerda $(10,0 \% \pm 5,5)$, cada vaso emite um ramo direto e indiviso em $3,33 \% \pm 3,3$. Em 3,33\% $\pm 3,33 \mathrm{a}$ a. torácica interna esquerda envia um ramo indireto e a subclávia esquerda um ramo direto, ambos indivisos; em $3,33 \% \pm 3,3$ a a. torácica interna esquerda fornece três ramos diretos e indivisos e a a. subclávia esquerda, um ramo, também direto e indiviso.

Quando a associação ocorre entre o tronco braquiocefálico $(6,66 \% \pm 4,5)$, em $3,33 \% \pm 3,3$ ambas enviam um ramo direto e indiviso; em $3,33 \% \pm 3,3$ a a. torácica interna esquerda fornece um ramo direto e outro indireto e o tronco braquiocefálico, um ramo direto, todos indivisos.

Quando a combinação se estabelece com as aa. torácica interna esquerda e subclávia esquerda $(16,66 \% \pm 6,8)$, cada vaso contribui com um ramo direto e indiviso em $3,33 \% \pm 3,3$; a a. torácica interna esquerda e a a. subclávia esquerda enviam um ramo direto, enquanto a torácica interna direita, um ramo indireto; em $3,33 \% \pm 3,3$ a a. torácica interna esquerda emite dois ramos diretos, a a. torácica interna direita um ramo indireto e a a. subclávia esquerda um ramo direto; em 3,33\% \pm 3,3 a a. torácica interna esquerda emite dois ramos indiretos e as outras duas, um ramo direto; em 3,33\% a a. torácica interna esquerda contribui com três ramos e as aa. torácica interna direita e subclávia esquerda com um ramo, todos diretos e indivisos.

Quando a associação ocorre com as aa. torácica interna direita e tronco braquiocefálico $(10,0 \% \pm 5,5)$, estas fornecem, por ordem, em $3,33 \% \pm 3,3$ dois ramos indiretos, um direto $\mathrm{e}$ outro indireto, todos indivisos; em $3,33 \% \pm 3,3$ a a. torácica interna esquerda emite dois ramos diretos, a a. torácica interna direita, um ramo indireto e do tronco braquiocefálico, um ramo direto, ambos indivisos; em 3,33\% $\pm 3,3$ a a. torácica interna esquerda contribui com dois ramos diretos e um indireto, a a. torácica interna direita, com um ramo direto e o tronco braquiocefálico com um ramo indireto, sendo todos indivisos.

Quando a a. torácica interna esquerda associa-se às aa. torácica interna direta e subclávia direta $(6,66 \% \pm 4,5)$, cada vaso contribui, em $3,33 \% \pm 3,3$, com um ramo direto e indiviso; em $3,33 \% \pm 3,3$ a a. torácica interna esquerda emite um ramo direto dividido em dois colaterais e um ramo indireto e indiviso, a a. torácica interna direita com um ramo indireto e indiviso e a a. subclávia direita com um ramo direto, também indiviso. 
DRUMMOND, S.S.; BOMBONATO, P.P.; SILVA, F.O.C.; SEVERINO, R.S.; SANTOS, A.L.Q.; MARIANA, A.N.B. Vascularização arterial da porção torácica do timo em suínos. Braz. J. vet. Res. anim. Sci. São Paulo, v.33, n.4, p.214-219, 1996.

\section{Tabela 1}

Número de vasos, diretos e indiretos, destinados à porção torácica do timo de suínos SRD, segundo sua origem e penetração no parênquima. São Paulo, 1992.

\begin{tabular}{c|c|c|c|c}
\hline NUMERO & \multicolumn{4}{|c}{ PENETRANTES } \\
\hline ORIGEM & \multicolumn{4}{|c}{} \\
\hline Obs. & diretos & indiretos & diretos & indiretos \\
\hline 1 & 6 & 2 & 6 & 2 \\
2 & 1 & 1 & 1 & 1 \\
3 & 2 & 2 & 2 & 2 \\
4 & 4 & 2 & 4 & 2 \\
5 & 2 & 2 & 2 & 2 \\
6 & 3 & 1 & 3 & 2 \\
7 & 3 & - & 3 & - \\
8 & 3 & - & 3 & - \\
9 & 2 & - & 2 & - \\
10 & 4 & - & 4 & - \\
11 & 2 & 1 & 2 & 1 \\
12 & 4 & 1 & 8 & 1 \\
13 & 3 & 2 & 3 & 2 \\
14 & 1 & 1 & 1 & 1 \\
15 & 2 & 1 & 2 & 1 \\
16 & 5 & 1 & 5 & 1 \\
17 & 5 & - & 5 & - \\
18 & 3 & 2 & 3 & 2 \\
19 & 3 & - & 3 & - \\
20 & - & - & - & - \\
21 & 3 & - & 3 & - \\
22 & 1 & 1 & 1 & 1 \\
23 & 2 & 2 & 3 & 2 \\
24 & 2 & - & 2 & - \\
25 & 2 & 1 & 2 & 1 \\
26 & 4 & 1 & 4 & 1 \\
27 & 3 & 1 & 3 & 2 \\
28 & 3 & 1 & 3 & 1 \\
29 & 2 & 1 & 2 & 1 \\
30 & 2 & 2 & 2 & 2 \\
\hline \hline Total & 82 & 29 & 87 & 31 \\
\hline
\end{tabular}

Quando a a. torácica interna esquerda associa-se com as aa. subclávia esquerda e tronco braquiocefálico $(3,33 \% \pm 3,3)$, elas emitem, respectivamente, um ramo indireto, um direto e outro também direto, todos indivisos.

Quando a associação se estabelece com o tronco braquiocefálico, a a. torácica interna direita e a a. subclávia esquerda $(6,66 \% \pm 4,5)$, em $3,33 \% \pm 3,3$, fornecem, por ordem, um ramo indireto, um direto e outro indireto e dois diretos, todos indivisos; em $3,33 \% \pm 3,3$ a a. torácica interna esquerda emite um ramo indireto, enquanto os outros vasos emitem um ramo cada um, diretos e indivisos.

Quando a a. torácica interna esquerda associa-se às aa. torácica interna direita, subclávia e tronco braquiocefálico, em $3,33 \% \pm 3,3$ esta envia dois ramos diretos e indiretos $e$ indivisos, e as outras emitem, respectivamente, um ramo direto e um direto, também indivisos.

Quando a associação ocorre com as aa. subclávia esquerda, torácica interna direita e tronco tireocervical, em $3,33 \% \pm 3,3$ a a. torácica interna esquerda contribui com três ramos diretos e indivisos, a subclávia esquerda com dois ramos diretos, um indireto e indivisos, a torácica interna direita, com um ramo indireto e o tronco tireocervical com ramo direto, ambos indivisos.

Quando ocorre a combinação com as aa. torácica interna direita, subclávia esquerda, subclávia direita e cervical superficial esquerda, em $3,33 \% \pm 3,3$ cada vaso emite um ramo, que, por ordem, são: direto, indireto, direto, direto, indireto e todos indivisos. Por fim, quando a irrigação é realizada exclusivamente pela a. torácica interna esquerda $(10,0 \% \pm 5,5)$, esta ocorre a partir de um ramo direto e um indireto, ambos indivisos em $16,66 \% \pm 6,8$; dois diretos e dois indiretos em $3,33 \% \pm 3,3$, também indivisos.

Independente da origem, do tipo das associações que possam ocorrer, um número variável de colaterais penetra nesta parte glandular, mais precisamente: dois - $16,66 \% \pm 6,8$; três $26,66 \% \pm 8,1$; quatro $-23,33 \% \pm 7,7$; cinco $-16,66 \% \pm 6,8 ;$ seis $-6,66 \% \pm 4,5$; oito $-3,33 \% \pm 3,3$ ou nove $-3,33 \% \pm 3,3$.

Finalmente, em relaçāo à ordenação dos vasos tímicos, considerando seu número, origem e tipo, notamos que não havia repetiçōes para cada uma das peças examinadas (Tab. 2).

\section{Sumário estatístico}

Analisando estatisticamente a participação dos diferentes vasos e seus tipos, que são responsáveis pela vascularização arterial do timo em suínos, pudemos evidenciar com auxílio do teste do qui-quadrado, com nível de significância de 5\%, que não existe diferença significativa quando confrontamos sexos e diferentes vasos participantes.

Ainda, utilizando-nos do Teste de Correlação de Pearson, com nível de significância de $1 \%$, encontramos $r=0,154841$ para a correlaçâo entre o número total de ramos na sua origem e o comprimento do animal e $r=0,216517$ para a correlação entre o número total de ramos que penetram no timo e o comprimento do espécime. Estes dados são indicativos de correlação positiva, porém com tendência à nulidade, ou seja, a correlação entre o número de vasos e o tamanho do animal está muito próxima de zero, evidenciando o fato de que as variáveis têm tendência muito pequena de indicar que o número de vasos aumenta na mesma relação do aumento do tamanho do animal estudado.

Finalmente, para facilitar as descrições das variáveis métricas, observadas para a porção torácica do timo, nós as tabulamos (Tab. 3). 
DRUMMOND, S.S.; BOMBONATO, P.P.; SILVA, F.O.C.; SEVERINO, R.S.; SANTOS, A.L.Q.; MARIANA, A.N.B. Vascularização arterial da porção torácica do timo em suínos. Braz. J. vet. Res. anim. Sci. São Paulo, v.33, n.4, p.214-219, 1996.

Tabela 2

ardenação dos ramos tímicos (em sentido caudocranlal) da sua porção torócica em suínos SRD, segundo a sua origem e tipo. São Paulo, 1992.

\begin{tabular}{|c|c|c|c|c|c|c|c|}
\hline \multirow[t]{2}{*}{ Obs. } & \multicolumn{7}{|c|}{ Ordenação dos vasos } \\
\hline & $1^{\circ}$ & $2^{\circ}$ & $3^{0}$ & $4^{\circ}$ & $5^{\circ}$ & $6^{\circ}$ & $7^{0}$ \\
\hline 1 & TIEd & TIEd & TREd & SCEd & SCEi & TIDi & $\pi \mathrm{Cd}$ \\
\hline 2 & TEI & TIEd & & - & - & - & - \\
\hline 3 & TBQd & TIEi & TEE & TIDd & - & - & - \\
\hline 4 & TBQd & TIEi & TIDd & TIDi & SCEd & SCEd & - \\
\hline 5 & TEEd & TEi & TREd & TEi & - & - & - \\
\hline 6 & TBQd & TIEd & TREd & $\pi \mathrm{Di}$ & - & - & - \\
\hline 7 & TEd & TIEd & TIDd & - & - & - & - \\
\hline 8 & TEEd & TIDd & SCDd & - & - & - & - \\
\hline 9 & TEEd & TIEd & - & - & - & - & - \\
\hline 10 & TIEd & ThEd & TREd & SCEd & - & - & - \\
\hline 11 & TBQd & TEi & SCEd & - & - & - & - \\
\hline 12 & TEd & $\mathrm{TDi}$ & $\mathrm{TIDd}$ & TEEd & - & - & - \\
\hline 13 & TEEd & TIDi & SCDd & SCEd & CSEi & - & - \\
\hline 14 & TEE & SCEd & - & - & - & - & - \\
\hline 15 & TEEd & TIEd & $\mathrm{TIDi}$ & - & - & - & - \\
\hline 16 & TBQd & TIEd & TTEd & TIDd & TEEd & SCDi & - \\
\hline 17 & ThEd & TIDd & TIEd & TIEd & SCEd & - & - \\
\hline 18 & TBQi & TIEI & TIEd & TREd & TIDd & - & - \\
\hline 19 & TIEd & TIEd & TIDd & - & - & - & - \\
\hline 20 & - & - & - & - & - & - & - \\
\hline 21 & TIEd & TIDd & SCEd & - & - & - & - \\
\hline 22 & $\mathrm{TEE}$ & TIEi & - & - & - & - & - \\
\hline 23 & TIEi & $\mathrm{TIDi}$ & TIEd & SCDd & - & - & - \\
\hline 24 & TBQd & TIEd & - & - & - & - & - \\
\hline 25 & TIEd & $\mathrm{TIDi}$ & SCEd & - & - & - & - \\
\hline 26 & TIEd & TIEd & TIEd & $\mathrm{TIDi}$ & TIDd & - & - \\
\hline 27 & TBOd & TIEi & TDd & SCEd & - & - & - \\
\hline 28 & TIEd & TEEd & $\mathrm{TDi}$ & SCEd & - & - & - \\
\hline 29 & TBOd & TIEi & TIEd & - & - & - & - \\
\hline 30 & TIEi & TEE & TDDd & SCEd & - & - & - \\
\hline
\end{tabular}

\begin{tabular}{|c|c|}
\hline CSD & a. cervical superficial direita \\
\hline CSE & a. cervical superficlal esquerda \\
\hline d & ramo direto \\
\hline 1 & ramo Indireto \\
\hline$S C D$ & a. subcláavia direita \\
\hline SCE & a. subclávla esquerda \\
\hline TBQ & tronco braqulocefálllco \\
\hline TD & a. toráclca interna direlta \\
\hline TE & a. torácica Interna esquerda \\
\hline$\pi \mathrm{C}$ & tronco a tireocervical \\
\hline
\end{tabular}

primeira sido objeto de nossos estudos. As nossas observações referentes ao órgāo indicaram-nos variabilidade na sua forma e no seu tamanho; assim é que a parte torácica do timo situou-se em posição dorso-ventral no mediastino cranial, à semelhança do ocorrido em outras espécies, como nos ruminantes.

Apesar de alguns autores, como Daschinger ${ }^{4}$ (1978) e Schummer et al. ${ }^{16}$ (1981), descreverem a existência de um istmo cervicotorácico e craniocervical, inclusive com irrigação própria, em nosso material nāo nos foi possível tal constatação, já que para as referidas porções citadas não notamos identidade anatômica, que em sua constituição, topografia, forma ou vascularização.

Ainda, relatos de autores como Schummer et al. ${ }^{16}$ (1981), estudando bovinos, e Wustinger; Pospieszny $^{19}$ (1984), estudando suínos, e que subdividiram a parte torácica em direita e esquerda, a nosso ver, carecem de exatidāo, pois pudemos observar que a massa tímica, chamada por eles de esquerda, avança para o antímero direito, podendo receber vasos oriundos e próprios deste antímero. Acrescente-se ainda o fato de que a massa tímica é toda ela constituída por lobos unidos por tecido conjuntivo, ficando difícil a indicação de uma faixa de tecido ou linha que una as duas metades, como comentam tais pesquisadores.

No que se refere à presença de um corpúsculo tímico acessório, como descrito por Bruni; Zimmerl $^{3}$ (1947), para os eqüídeos, cabe-nos assinalar que em nosso material tal achado não pôde ser confirmado. Nossa opinião é que a referida citação talvez tenha sido feita na decorrência da variabilidade de forma e tamanho que o timo apresenta, particularmente em algumas espécies.

Das observações constantes na revisão da literatura podemos inferir que as informações prestadas pelos tratadistas clássicos, tais como Martin $^{12}$ (1912), Martin; Schauder ${ }^{13}$ (1938) e Ellenberger; Baum 6 (1977), são na maioria das vezes imprecisas, fato este próprio do objetivo dos tratados, que indicam conhecimentos genéricos. Entretanto, os dados relatados a respeito de determinada espécie, como se fossem verdadeiros para outras, merecem, de nossa parte, crítica, já que a simples observação macroscópica do timo

\section{DISCUSSĀO}

O timo dos suínos foi considerado, para fins de descrição dos resultados, como constituído de duas partes, a torácica (pars thoracica) e a cervical (pars cervicales), tendo apenas a mostra ser este órgão uma estrutura de aspectos e características extremamente variados, o que dificulta ou mesmo impede a generalização de conhecimentos feita pelos autores. Ainda que incompletos, a nosso ver, os relatos constantes destes livros merecem comparação. 
DRUMMOND, S.S.; BOMBONATO, P.P.; SILVA, F.O.C.; SEVERINO, R.S.; SANTOS, A.L.Q.; MARIANA, A.N.B. Vascularizaçāo arterial da porçāo torácica do timo em suínos. Braz. J. vet. Res. anim. Scl. São Paulo, v.33, n.4, p.214-219, 1996.

Tabela 3

Sumário estatístico das varí́vels métricas referentes ao número de ramos tímicos endereçados à porção tord́cica, em suínos SRD, segundo sua origem e tipo. São Paulo, 1992.

\begin{tabular}{c|c|c|c|c|c|c}
\hline \multirow{2}{*}{ TIPO } & \multicolumn{3}{|c|}{ ORIGEM } & \multicolumn{3}{|c}{ PENETRANTES } \\
\cline { 2 - 7 } & direto & indireto & total & direto & indireto & total \\
\hline VARIAVEL & & & & & & \\
média & 2,73 & 0,96 & 3,7 & 2,9 & 1,03 & 3,93 \\
mediana & 3 & 1 & 4 & 3 & 1 & 4 \\
mínimo & 0 & 0 & 0 & 0 & 0 & 0 \\
máximo & 6 & 2 & 8 & 8 & 2 & 9 \\
\hline
\end{tabular}

\section{Origem dos vasos tímicos}

Destacamos a descrição de Schummer et al. ${ }^{16}$ (1981) a respeito da origem dos vasos tímicos. $\mathrm{O}$ autor indica um maior número de vasos que fornecem ramos tímicos, relatando, também, a participação do tronco braquiocefálico, e da a. torácica interna esquerda, fato que foi também por nós observado. Ressalta-se ainda que este autor refere que tais vasos irrigam o timo nos bovinos, similar ao dos suínos.

Os outros autores, como Favilli ${ }^{7}$ (1943), Bruni; Zimmer $^{3}$ (1947), Koch ${ }^{9}$ (1963), Schwarze; Schröder ${ }^{17}$ (1972) e Getty ${ }^{8}$ (1975), relatam vasos como as aa. carótidas comum, subclávia, torácica interna, tronco braquiocefálico, na irrigação do timo, fato diferente daquele por nós observado.

Quanto à literatura especializada na vascularização do timo, autores, como Deniz' (1964), Luckhaus ${ }^{10}$ (1966) e Bombonato et al. ${ }^{1,2}(1986,1988)$, que realizaram suas pesquisas em fetos de bovinos azebuados e de origem européia, relatam a presença de ramos provenientes do tronco braquiocefálico, e das aa. torácica interna esquerda e direita torácica externa esquerda.

Confrontando com nossos resultados, apesar de serem animais de espécies diferentes, não verificamos a presença da a. torácica externa esquerda. Entretanto, encontramos ramos das á. subclávia esquerda e direita e cervical superficial esquerda e direita, fato não identificado pelos autores.

Em relação às pesquisas de Machado ${ }^{11}$ (1989), que estudou fetos de eqüinos SRD, nossos achados são ainda mais diferentes, como o relato da presença de ramos da a. pericárdica na irrigação da porção torácica do timo, não identificada em nenhum de nossos casos.

Silva $^{18}(1989)$ e Santos ${ }^{15}$ (1990), que estudaram fetos de caprinos SRD e ovinos SRD, respectivamente, são os que mais se aproximam, entre as outras espécies que não a por nós estudada, tanto na similaridade morfológica do timo, como nos vasos encontrados e no número de ramos colaterais destes vasos que suprem o órgão. Apesar disso, estes autores identificaram ramos do tronco bicarotídeo $\mathrm{e}$ tronco costocervical esquerdo na vascularização da porção torácica do órgão, fato por nós nunca encontrado. Por outro lado, na porção torácica, encontramos ramos da a. subclávia direita não identificados por aqueles autores.
Ao confrontarmos nossos resultados com aqueles obtidos nos trabalhos específicos com suínos, como o de Daschinger ${ }^{4}$ (1978), notamos algumas diferenças, já que o autor afirma ser a parte torácica do timo aquela que recebe apenas ramos diretos das aa. torácica interna direita e esquerda, do tronco braquiocefálico e da a. subclávia esquerda. No entanto, além dos vasos citados, notamos também a participação das aa. subclávia direita, cervical superficial esquerda e tronco tireocervical, em número maior de ramos, sendo estes ramos tanto diretos como indiretos, e ainda por vezes dividindo-se antes de alcançar o parênquima do órgāo.

Quando justapomos nossos achados com os de Wustinger; Pospieszny ${ }^{19}(1984)$, verificamos algumas semelhanças tanto na vascularização como na divisão do timo. Assim é que o autor relata que a porção torácica recebe ramos oriundos das aa. torácica interna e pericardicofrênica direita e esquerda, esta última não observada em nenhuma de nossas peças. No entanto, constatamos a presença de ramos do tronco braquiocefálico e das aa. subclávia direita e esquerda, não encontradas pelos autores.

\section{Típo e número de vasos}

Ao considerarmos o número de ramos destinados ao timo em suínos, identificamos em nosso trabalho um número total superior aos encontrados por Wustinger; Pospieszny ${ }^{19}$ (1984); Bombonato et al. ${ }^{1,2}(1986,1988)$; Machado ${ }^{11}$ (1989); Silva ${ }^{18}$ (1989) e Santos ${ }^{15}$ (1990), relativamente às espécies pesquisadas por estes autores, fato que pode ter sua explicação baseada no tamanho e na forma do timo, nos suínos. Vale frisar que a simples comparação numérica tem por objetivo a caracterização das variáveis com que o órgão se apresenta nas diferentes espécies, impedindo as freqüentes generalizaçōes dos conhecimentos obtidos em uma ou outra espécie para o conjunto dos animais domésticos, como fazem alguns autores.

Com relação ao tipo de vasos que penetram no timo, não notamos na literatura compulsada menção ou distinção ao fato.

Um fato importante, e que se repetiu neste experimento, foi o de que, quando considerados conjuntamente o número, a origem e a ordenação dos vasos tímicos, cada preparação apresentou individualidade característica, o que pode sugerir outras colocações de ordem embriológica na caracterização deste órgão, e que devem merecer atenção em outros trabalhos específicos.

No que se refere aos estudos estatísticos, ressalte-se a tendência à nulidade em relação ao tamanho do feto e número de vasos, indicando que o tamanho do feto, se interfere, o faz de maneira muito tênue quando relacionado ao aumento do número de vasos. 


\section{SUMMARY}

The systematization of the thymic vessels was analysed in 30 foetuses and newborn pigs. These vessels were dissected after injection of colored Neoprene Latex 450 . The thymus thoracic portion was directly or indirectly irrigated by branches from the trunk braquiocephalic, subclavians, internal thoracics, superficial cervical arteries, as well as from the thyreocervical trunk. The total number of collateral vessels varied from two to nine, and three the most frequently found.

UNITERMS: Anatomy; Pigs; Thymus, glands; Vascularization; Arteries.

\section{REFERÊNCIAS BIBLIOGRÁFICAS}

1-BOMBONATO, P.P.: BORELLI, V.; DIDIO, L.J.A. Arterial supply of the zebu fetuses. Archivio Italiano di Anatomia e di Embriologia. v.93, n.1, p.55-66, 1988.

2-BOMBONATO, P.P.: BORELLI, V.: FERNANDES FILHO, A. Contribuição ao estudo da vascularização arterial em fetos de bovinos de origem européia Revlsta da Faculdade de Medicina Veterinária e Zootecnia da Unlversidade de São Paulo, v.23, n. 1, p.31-8, 1986.

3-BRUNI, A.C.; ZIMMERL, U. Anatomia degli animali domesticl. Milano, Francesco Vallardi. 1947, v.2 p.257-61.

4-DASCHINGER, E. Topographic und Vaskularisation des Schweinethymus beim neugeborenen und drei Wochen alten Ferkel. München, 1978. 72p. Inaugural-Dissertation.

5-DENIZ, E. Die Blutegefabeersongung des Thymus beim Kalb. Zentralblat fur Veterinarmedizin, A, v.11, p.750-8, 1964 .

6-ELLENBERGER. W.: BAUM, H. Handbuch der vergleichenden Anatomie der Haustiere, 18. auf. Berlin, Springer, 1977, p.602-4.

7-FAVILLI, N. Nozione comparate di anatomia e fisiologia degli animali agricoli. Milano, Francesco Vallardi, 1943. p.423.

8-GETTY, R. Sisson and Grossmann's the anatomy of the domestic animals. 5.ed. Philadelphia, W.B. Saunders, 1975. v.2, p.1273.

9-KOCH, T. Lehrbuch der Veterinar-Anatomie, Jena, Gustav Fischer, 1963, v.2, p.16.

10-LUCKHAUS, G. Die Pars cranialis thymi beim fetalen Rind. Zentralblat Pur Veterinarmedizin, A, v.13, p.414-27, 1966.

11-MACHADO, G.V. Sobre a origem, o número e a esqueletopia dos ramos arteriais destinados ao timo, em fetos de eqüinos SRD. São Paulo, 1989. 30p. Tese (Doutorado) - Faculdade de Medicina Veterinária e Zootecnia, Universidade de Sāo Paulo.

12-MARTIN, P. Lehrbuch der Anatomie der Haustlere, Stuttgart, Verlag von Schickhardt \& Ebner, 1912. v.1, p.430.

13-MARTIN, P.; SCHAUDER, N. Lehrbuch der Anatomie der Haustiere. Stuttgart, Schickhardt \& Ebner, 1938. v.3, p.370.

14-NOMINA ANATOMICA VETERINARIA, revised by the International Committee on Veterinary Gross Anatomical Nomenclature. 3.ed. Ithaca, World Association of Veterinary Anatomists, 1983. 209 p.

15-SANTOS JUNIOR, I. Suprimento arterial do timo em ovinos sem raça definida. São Paulo, 1990. 34p. Dissertação (Mestrado) - Faculdade de Medicina Veterinária e Zootecnia, Universidade de São Paulo.

16-SCHUMMER, A.; WILKENS, H.; VOLLMERHAUS, B.; HABERMEHL, K.H The anatomy of the domestic animals. Berlin, Paul Perey, 1981, p.283-8.
17-SCHWARZE, E.; SCHRÖDER, L, Compendio de anatomía veterlnaria. Zaragoza, Acribia, 1972, v.3. p.180-2.

18-SILVA, F.O.C. Suprimento arterial do timo em fetos de caprinos SRD. São Paulo, 1989. 44p. Tese (Doutorado) - Faculdade de Medicina Veterinária c Zootecnia, Universidade de São Paulo.

19-WUSTINGER, J.; POSPIESZNY, N. Die arterielle Vaskularisation des Schweinethymus in de zweiten Halfte der pranatalen Periode. Zentralblat fur Veterinarmedizin, C, v.13, p.341-50, 1984. 